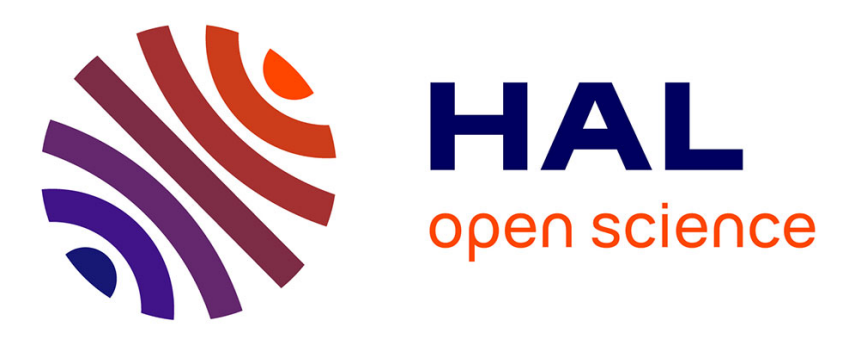

\title{
On the incorporation of surface tension in finite-element calculations
}

Jean-Baptiste Leblond, Hussein Amin El Sayed, Jean-Michel Bergheau

\section{To cite this version:}

Jean-Baptiste Leblond, Hussein Amin El Sayed, Jean-Michel Bergheau. On the incorporation of surface tension in finite-element calculations. Comptes Rendus Mécanique, 2013, 341 (11-12), pp.770

- 775. 10.1016/j.crme.2013.10.004 . hal-01433986

\section{HAL Id: hal-01433986 \\ https://hal.sorbonne-universite.fr/hal-01433986}

Submitted on 13 Jan 2017

HAL is a multi-disciplinary open access archive for the deposit and dissemination of scientific research documents, whether they are published or not. The documents may come from teaching and research institutions in France or abroad, or from public or private research centers.
L'archive ouverte pluridisciplinaire HAL, est destinée au dépôt et à la diffusion de documents scientifiques de niveau recherche, publiés ou non, émanant des établissements d'enseignement et de recherche français ou étrangers, des laboratoires publics ou privés. 


\title{
On the incorporation of surface tension in finite element calculations
}

\author{
Jean-Baptiste Leblond $^{\mathrm{a}}$, Hussein Amin El Sayed ${ }^{\mathrm{b}}$, Jean-Michel Bergheau ${ }^{\mathrm{b}}$ \\ ${ }^{a}$ UPMC Univ Paris 06, UMR 7190, Institut Jean Le Rond d'Alembert, Tour 65-55, 4 place Jussieu, 75252 Paris Cedex \\ 05, France \\ ${ }^{\mathrm{b}}$ Université de Lyon, ENISE, LTDS, UMR 5513 CNRS, 58 rue Jean Parot, 42023 Saint-Etienne Cedex 02, France \\ Received *****; accepted after revision +++++
}

\begin{abstract}
The aim of this paper is to propose a new method for introduction of surface tension effects (including both the "curvature effect" and the "Marangoni effect") into finite element computations. Usual methods consider the loads induced by surface tension onto the fluid. We propose to instead directly model surface tension itself through introduction into the mesh of surfacic elements having a planar hydrostatic state of stress, and representing a fictitious tight membrane stuck onto the interface. Such a method is very easy to program and very efficient. Two numerical examples are provided to illustrate this efficiency. The first pertains to a problem of small oscillations of a liquid due to combined gravity and surface tension, and illustrates the effect of the normal component of the surface tension load (curvature effect). The second pertains to a problem involving a surfacic gradient of temperature, and illustrates the effect of the tangential component of the surface tension load (Marangoni effect).
\end{abstract}

Key words: Surface tension; finite element method; membrane elements

\section{Introduction}

Introducing surface tension effects into simulations of fluid flows based on the finite element method is unavoidable for some applications. For instance, if one wants to perform a complete simulation of a welding operation incorporating both the solid and liquid phases, the presence of the solid imposes the choice of the finite element method; on the other hand the fluid flow in the molten pool is very sensitive to surface tension, which leaves no choice but to include it in the simulation.

Email addresses: jbl@lmm.jussieu.fr (Jean-Baptiste Leblond), aminelsa@enise.fr (Hussein Amin El Sayed), bergheau@enise.fr (Jean-Michel Bergheau). 
The influence of surface tension on the fluid flow arises from two effects: (i) it generates a normal force onto the free surface of the fluid, proportional to the curvature of this surface ("curvature effect"); (ii) when it is non-uniform (for instance as a result of a non-uniform temperature field), its gradient generates an additional tangential force onto the free surface ("Marangoni effect"). In previous implementations of surface tension in finite element programmes (Ruschak [1], Bach and Hassager [2], Christodoulou and Scriven [3], Cairncross et al. [4], Bellet [5], Dettmer et al. [6], Rabier and Medale [7], Dettmer and Peric [8], Saksono and Peric [9]), these two effects were accounted for in a natural way, by including the loads (in fact, generally the sole normal one) applied on the free surface of the fluid among the external forces of the problem.

In such an approach incorporation of the tangential force (Marangoni's effect) does not raise any particular issue. However that of the normal force (curvature effect) is more difficult. Indeed direct calculation of this force, like in [5] and [7], requires an evaluation of the mean curvature (trace of the curvature tensor) of the free surface. But the position of this surface depends on the motion of the fluid, so that calculation of its curvature demands an evaluation of the second spatial derivatives of the displacement field. This may be done by using elements of class $\mathcal{C}^{1}$, but such elements are very cumbersome in $2 \mathrm{D}$ and $3 \mathrm{D}$ and as a rule not available in standard finite element codes. Another solution consists of using classical elements of class $\mathcal{C}^{0}$ and employing some "trick" to get the mean curvature; a good one, for instance, consists of evaluating it from the divergence of the normal to the free surface using the nodal values of this normal and the first derivatives of the shape functions, these nodal values being themselves estimated by averaging the normals to the elements containing the node considered. But the calculation of curvature may then involve significant errors having a negative impact on the accuracy of the results.

Another less direct, but more efficient approach, used in $[1,2,3,4,6,8,9]$, consists in integrating by parts that term of the weak formulation of the problem corresponding to the virtual power of the normal force exerted onto the free surface. The major advantage is the total disappearance of the second spatial derivatives of the displacement field from the weak formulation, which permits to use standard elements of class $\mathcal{C}^{0}$ with no particular difficulty. But in the papers just mentioned this approach remained incomplete in that it included only the curvature effect and forgot about the Marangoni effect; and furthermore the weak formulation contained an error in the form of a spurious integral over the bounding line of the free surface, as will be detailed below.

The aim of this paper is to propose a new, very simple and efficient method of incorporation of surface tension effects into finite element calculations, based on the well-known equivalence of the effects of surface tension and those of a fictitious tight membrane stuck onto the interface. The idea is thus to avoid introducing surface tension through the loads it induces onto the free surface of the fluid, but to instead directly introduce surface tension itself through incorporation into the mesh of surfacic membrane elements having a planar hydrostatic state of stress. The transmission of forces from the "numerical membrane" to the fluid is ensured by the weak formulation of the problem itself. This formulation is basically similar to that obtained by the approach expounded in $[1,2,3,4,6,8,9]$, but now includes the Marangoni effect and is free of any spurious line integral.

The paper is organized as follows:

- In Section 2, for completeness and clarity, we recall the classical equivalence just mentioned, for both the normal and tangential components of the force exerted onto the free surface of the fluid.

- In Section 3, we consider a problem involving only a normal force (curvature effect), and compare the results of the new numerical method to those obtained through direct computation of this force via the mean curvature of the free surface. The aim here is to illustrate the superiority of the new approach for such a case.

- In Section 4, we do the same thing but for a problem involving only a tangential force (Marangoni's effect). Since the new method does not have any major advantage over previous ones for such a case, the aim there is simply to show that it provides equivalent results. 


\section{Modelling surface tension through surfacic membrane elements}

Physics teaches us that the effect of surface tension is equivalent to that of a fictitious tight membrane stuck onto the interface between the two media in contact. The following two figures illustrate this in a $2 \mathrm{D}$ context.

Figure 1 first shows the equivalence for the normal component of the surface tension load. In this figure, the fluid is assumed to lie for instance below the gas, and the interface is supposed to be curved toward the fluid, with a radius of curvature $R$. A portion of elementary length $d s$ of the fictitious membrane is materialized by thick lines. The two forces exerted on the left and right sides of this portion by the rest of the membrane have equal intensities $\gamma$ (the surface tension) but non-parallel directions, because of the curvature of the interface. As a result, they are not equilibrated; equilibrium of the portion of membrane considered demands that a vertical upward force (in full line in the figure) $2 \alpha \gamma$ be exerted on it by the fluid, where $\alpha$ is the angle shown in the figure. This angle is obviously equal to $\frac{1}{2} \frac{d s}{R}$; hence the value of the force is $\frac{\gamma}{R} d s$. The action-reaction law implies that the membrane must exert a vertical downward force (in dotted line in the figure) on the fluid, of intensity $\frac{\gamma}{R}$ per unit length of the interface. Now this is precisely the classical expression of the normal load exerted on the surface of a fluid, as a result of surface tension effects; this demonstrates the equivalence.

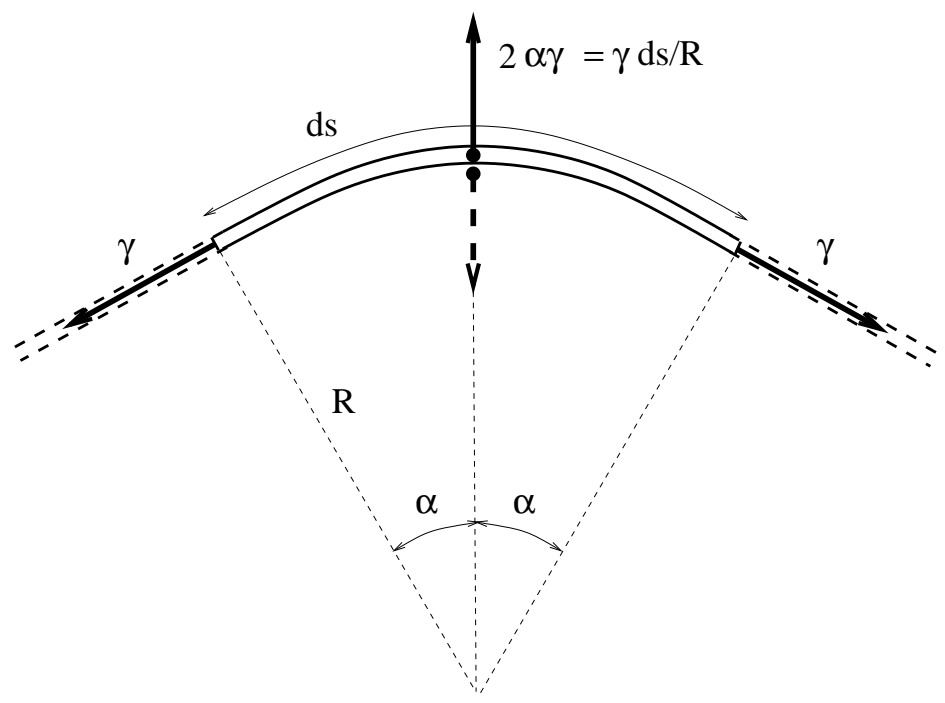

Figure 1. Effect of surface tension on a curved interface

Figure 2 now shows the equivalence for the tangential component of the surface tension load. The fluid is again assumed to lie below the gas. The interface is supposed here to be flat for simplicity, but the surface tension is allowed to vary along it. A portion of the fictitious membrane of length $d x$ is again materialized by thick lines. The forces exerted on the left and right sides of this portion by the rest of the membrane are $-\gamma$ and $\gamma+d \gamma$ respectively; these forces are not equilibrated so that equilibrium of the portion of membrane demands that a horizontal force (in full line in the figure) $-d \gamma=-(d \gamma / d x) d x$ be exerted on it by the fluid. By the action-reaction principle, the membrane must exert a tangential force (in dotted line in the figure) on the fluid, of intensity $d \gamma / d x$ per unit length of the interface. Now this is again exactly the classical expression of the tangential load exerted on the surface of a fluid, as a result of the gradient of surface tension (Marangoni's effect); this again demonstrates the equivalence. 


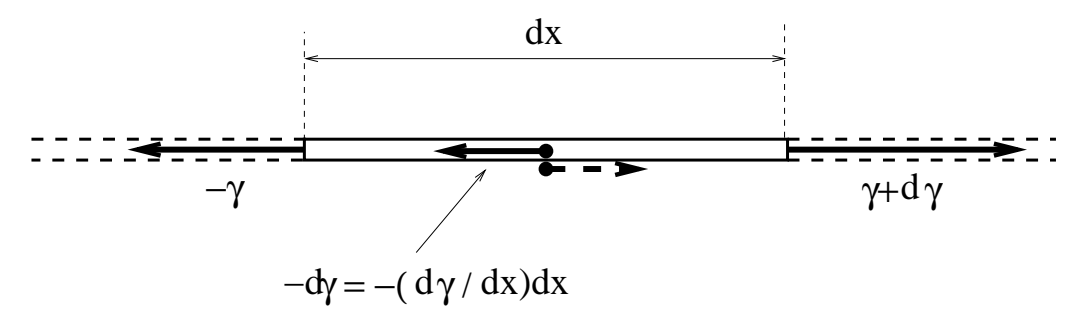

Figure 2. Effect of a gradient of surface tension on a flat interface (Marangoni's effect)

Advantage may be taken of the equivalence from a numerical point of view. Indeed, instead of accounting for surface tension effects through the loads they induce on the fluid, like in standard approaches $[1,2,3,4,5,6,7,8,9]$, one may directly incorporate the fictitious tight membrane into the finite element mesh, thus giving it a kind of "numerical", if not "physical" existence. This means in practice introducing new surface elements whose nodes coincide with those of the 3D mesh located on the free surface, having a surfacic hydrostatic state of stress $\boldsymbol{\sigma}$ given by

$$
\boldsymbol{\sigma}=\gamma \mathbf{1}_{S}
$$

where $\gamma$ denotes the surface tension like above and $\mathbf{1}_{S}$ the local surfacic $2 \mathrm{D}$ unit tensor. This state of stress is then naturally accounted for through the famous term " $\int{ }^{t} \mathbf{B} . \boldsymbol{\sigma}$ " appearing classically in the weak formulation of the problem. In this approach, the transmission of forces from the membrane to the fluid is a result of (i) the fact that the membrane is stuck onto the fluid since its nodes coincide with those of the 3D mesh lying on the free surface; and (ii) the correct expression of the virtual power of internal forces including the surfacic tension of the membrane, through the term $\int{ }^{t} \mathbf{B} . \boldsymbol{\sigma}$.

From the programming point of view, putting the idea to practice is very simple since it just requires the development of special surface elements with state of stress given by equation (1). Also, it eliminates the need for evaluation of the mean curvature of the free surface, and therefore for calculation of the second spatial derivatives of the displacement field.

In fact, the weak formulation of the problem obtained in this way is basically similar to that deduced in $[1,2,3,4,6,8,9]$ from multiplication of the strong equations of the problem by test functions and integration by parts of the surface integral pertaining to surface tension effects. However the new formulation is free of the shortcomings of the former one:

(i) The authors of $[1,2,3,4,6,8,9]$ considered the sole case of a uniform surface tension within the interface, exerting only a normal force onto the fluid, without apparently noticing that their approach also permitted to account for the tangential force (Marangoni's effect) with only little additional effort.

(ii) The former weak formulation involved an extra integral over the line $\mathcal{L}$ bounding the free surface, the presence of which could easily be seen to necessarily result from an error. Indeed this line integral could only represent a portion of either the virtual power of external forces, or the virtual power of internal forces. Now two cases could be envisaged. If the line $\mathcal{L}$ was in contact with a medium exerting external forces on it, these external forces had to include an "adhesion force" normal to the surface of this medium; but no term corresponding to such a force appeared in the formulation of $[1,2,3,4,6,8,9]$. If, on the other hand, no external forces were applied on $\mathcal{L}$, the integral over this line could but represent the virtual power of some internal forces; but then the expression of the virtual power of all internal forces was such that it did not vanish in rigid-body motions.

In fact, the origin of the error could easily be traced back to an incomplete assessment of the forces exerted onto the bounding line $\mathcal{L}$. Indeed the force applied on it by the membrane had to be balanced by forces exerted either by other membranes (arising from other interfaces), or by the 
surrounding 3D phases, or by the external medium; none of which was included in the formulation of $[1,2,3,4,6,8,9]$. Incorporation of these extra forces would have introduced terms exactly compensating the line integral.

\section{Numerical example for the curvature effect}

To illustrate the application of the method proposed, we first consider a case where the surface tension is uniform within the interface. The problem envisaged, identical to that considered by Rabier and Medale [7], is that of small oscillations of an incompressible fluid having a free upper surface; the oscillations are due to both gravity and surface tension effects on this surface, and are dampened by viscosity. The fluid is enclosed in a parallelepipedic box and allowed to move freely along its lateral sides; the problem is in fact $2 \mathrm{D}$ in a vertical plane. Its analytic solution was provided by Prosperetti [10].

The width, height and thickness of the box are $1 \mathrm{~m}, 1 \mathrm{~m}$ and $0.4 \mathrm{~m}$ respectively. The upper free surface is initially perturbed into a sinusoid of wavelength equal to twice the width of the box and amplitude $0.01 \mathrm{~m}$. The physical constants are as follows: gravity, $g=1 \mathrm{~ms}^{-2}$; mass per unit volume, $\rho=1 \mathrm{~kg} \mathrm{~m}^{-3}$; kinematic viscosity, $\nu=0.01 \mathrm{~m}^{2} \mathrm{~s}^{-1}$; surface tension $\gamma$, between 0.001 and $1 \mathrm{Nm}^{-1}$. The values of $g$ and $\rho$ here are arbitrary, while the minimum and maximum values of $\gamma$ are adjusted so as to make surface tension effects negligible with respect to, or conversely dominating over, those of gravity.

We first show a result obtained by incorporating surface tension through the loads induced onto the fluid; in this version of the programme the normal force is evaluated from the mean curvature of the free surface, this curvature being deduced from the divergence of the normal to this surface, obtained itself through some approximate procedure. Figure 3 thus shows the distribution of the vertical displacement on the deformed mesh obtained at a typical instant of the calculation. (In this figure the distortion of the initial mesh is amplified by a factor of 20 for clarity, but the scale of the arrows representing the displacements is arbitrary). The aim here is to show that this distribution, although qualitatively reasonable, is not smooth enough to permit an accurate evaluation of the second derivative of the velocity or displacement fields, indispensable for a precise determination of the normal force. As a result, such simulations give very inaccurate results disagreeing with Prosperetti's analytical solution. (It is worth noting however that Rabier and Medale [7] obtained an acceptable solution of this problem by the same method but with a considerably more refined mesh, and a quadratic instead of linear interpolation scheme).

We now show results obtained by incorporating tight membrane elements into the mesh. Figure 4 compares the maximum vertical displacement of the free surface obtained numerically, as a function of time, to that provided by Prosperetti's solution. A very good agreement of the numerical and theoretical solutions may be observed, even when the period of oscillations arising from gravity alone (red curve in the figure) is reduced by as much as a factor of 3 by surface tension effects (violet curve in the figure).

\section{Numerical example for the Marangoni effect}

We now consider a case where the surface tension varies along the interface, giving thus rise to Marangoni's effect; in contrast the normal force is eliminated by prescribing a zero normal velocity on the flat free surface. Even though, the problem envisaged is a complex one, involving a parallelepipedic box containing a fluid the upper surface of which is heated non-uniformly; the resulting non-uniformity of the surface tension generates, via Marangoni's effect, a vortex-like motion of the fluid. No analytical solution is available for this problem, but one may compare the solutions obtained by incorporating surface tension in the standard way, through the loads induced onto the fluid, and the new one, through incorporation 


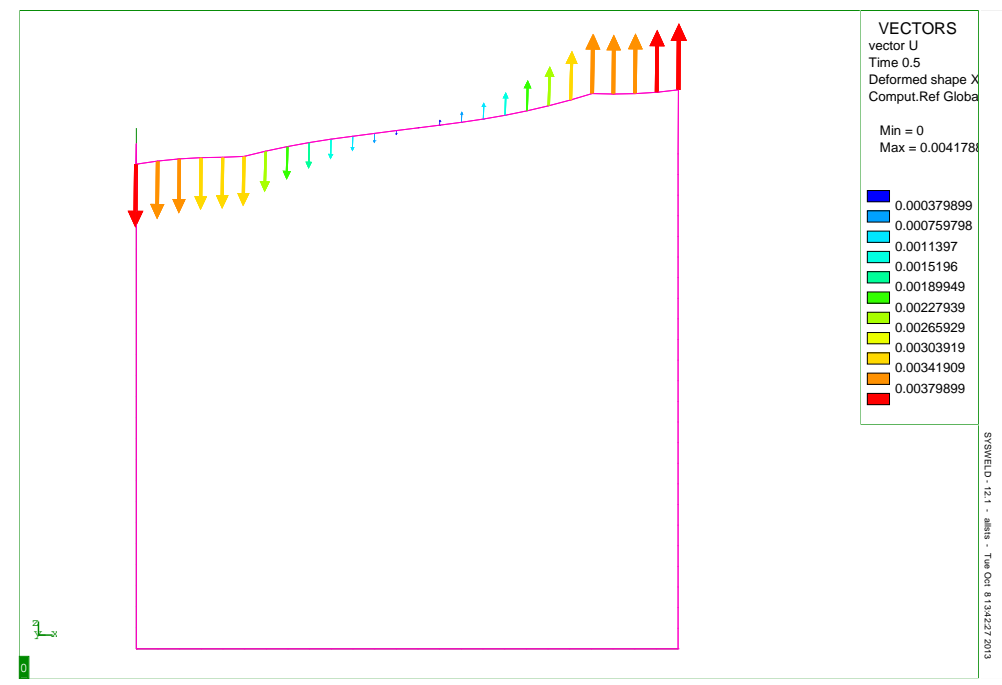

Figure 3. Typical displacement field in the oscillation problem - Surface tension accounted for through the induced load

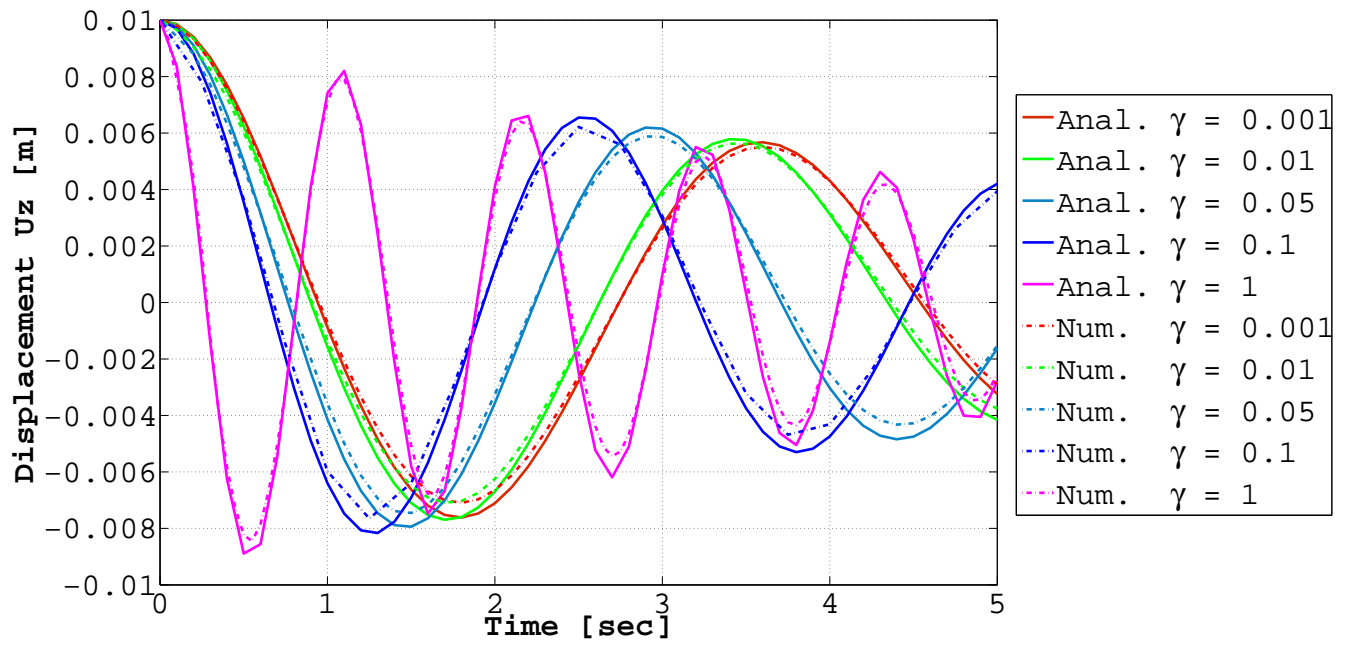

Figure 4. Time oscillations of a fluid arising to gravity and surface tension - Surface tension accounted for through membrane elements $\left(\gamma\right.$ in $\left.\mathrm{Nm}^{-1}\right)$

of tight membrane elements into the mesh. Note that the calculation of these loads does not raise any special problem here since the evaluation of the mean curvature of the (flat) interface is not required.

Figure 5 compares the velocity fields at a typical instant obtained in the two simulations. The agreement may be observed to be excellent. 


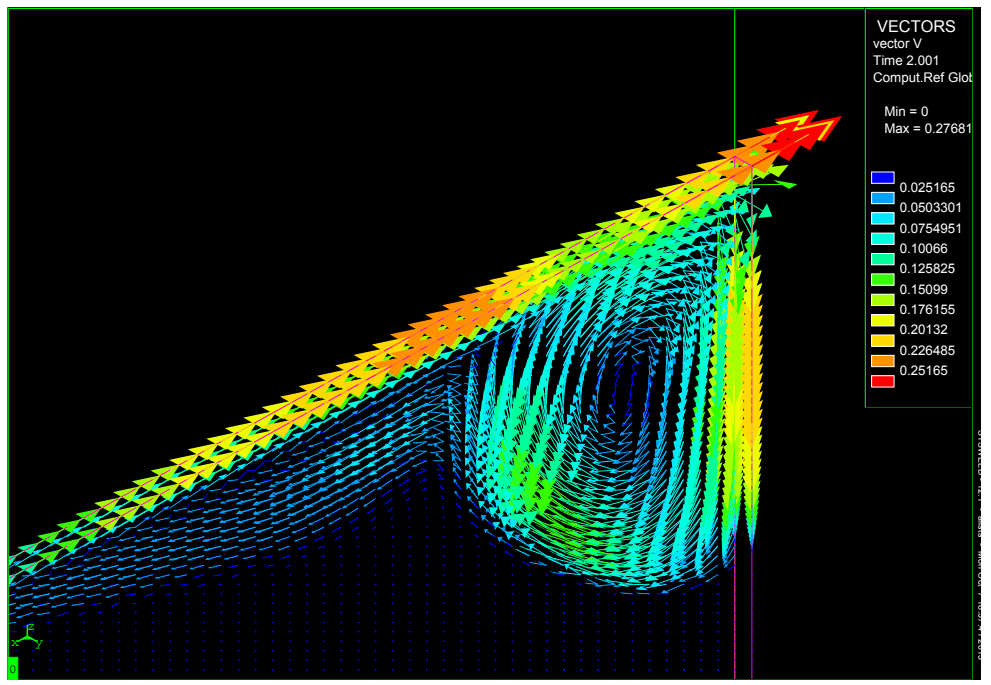

(a) Surface tension accounted for through the induced load

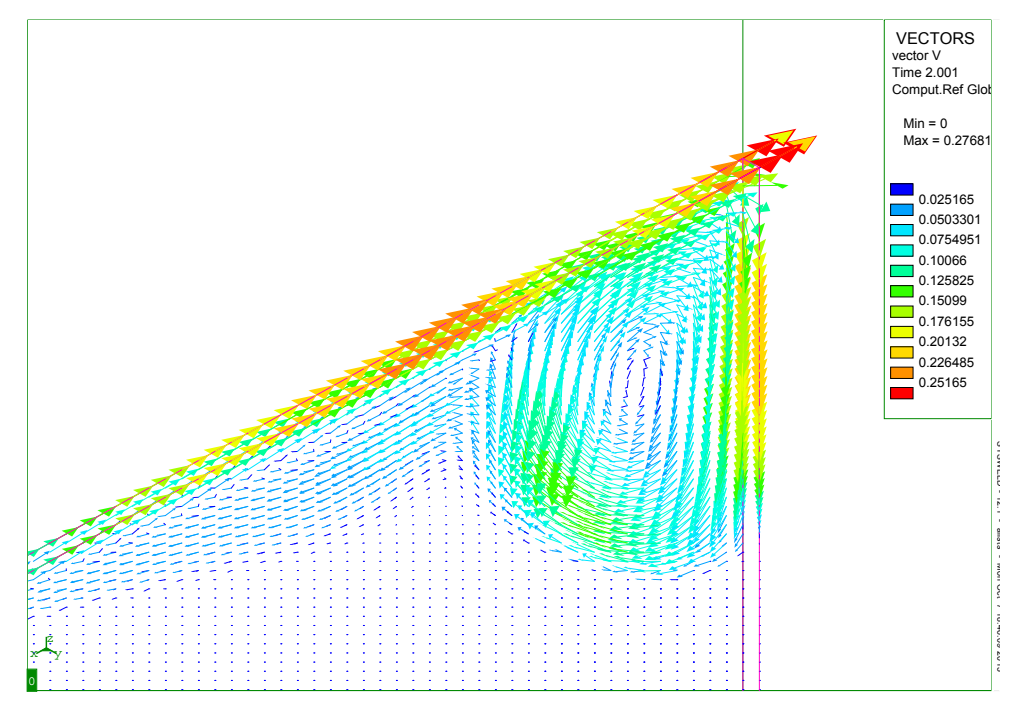

(b) Surface tension accounted for through membrane elements

Figure 5. Velocity fields induced by Marangoni's effect

\section{Conclusion}

The aim of this paper was to propose a new method for incorporation of surface tension effects in finite element computations. Whereas classical approaches account for surface tension through the loads 
it induces onto the fluid, the new one directly incorporates it through introduction of a fictitious tight membrane into the mesh, using the well-known equivalence between the effects generated by such a membrane and those of surface tension. This new method incorporates both the normal force exerted onto the free surface (curvature effect) and the tangential one (Marangoni effect) in a very simple and efficient way.

Practical application of the method was illustrated through two examples. In the first one, the surface tension exerted only a normal force onto the surface of the fluid (curvature effect). An approach based on direct calculation of this normal force through evaluation of the curvature of the free surface was found to fail (at least for the crude discretization and interpolation scheme used) because of inaccuracies in this evaluation, whereas the approach based on introduction of a tight membrane into the mesh was found to provide results agreeing very well with Prosperetti's analytical solution [10].

In the second example, the force exerted onto the surface of the fluid, arising from a nonzero gradient of the surface tension along the interface, was purely tangential (Marangoni's effect), thus making the test much less critical for the classical approach. The problem considered was too complex to be amenable to some analytic solution, but comparison between the results obtained with the classical and new methods revealed an excellent agreement between the two.

\section{References}

[1] K.J. Ruschak, A method for incorporating free surface boundaries with surface tension in finite element fluid-flow simulators, Int. J. Numer. Meth. Engng. 15 (1980) 639-648.

[2] P. Bach, O. Hassager, An algorithm for the use of the Lagrangian specification in Newtonian fluid mechanics with applications to free surface fluid flow, J. Fluid Mech. 152 (1985) 173-190.

[3] K.N. Christodoulou, L.E. Scriven, The fluid mechanics of slide coating, J. Fluid Mech. 208 (1989) 321-354.

[4] R.A. Cairncross, P.R. Schunk, T.A. Baer, R.R. Bao, P.A. Sackinger, Finite element method for free surface flows of incompressible fluids in three dimensions. Part I. Boundary fitted mesh motion, Int. J. Numer. Meth. Fluids 33 (2000) $375-403$.

[5] M. Bellet, Implementation of surface tension with wall adhesion effects in a three-dimensional finite element model for fluid flow, Comm. Numer. Meth. Engng. 17 (2001) 563-579.

[6] W. Dettmer, P.H. Saksono, D. Peric, On a finite element formulation for incompressible Newtonian fluid flows on moving domains in the presence of surface tension, Comm. Numer. Meth. Engng. 19 (2003) 659-668.

[7] S. Rabier, M. Medale, Computation of free surface flows with a projection FEM in a moving mesh framework, Comput. Meth. Appl. Mech. Engng. 192 (2003) 4703-4721.

[8] W. Dettmer, D. Peric, A computational framework for free surface fluid flows accounting for surface tension, Comput. Meth. Appl. Mech. Engng. 195 (2006) 3038-3071.

[9] P.H. Saksono, D. Peric, On finite element modelling of surface tension. Variational formulation and applications - Part I: Quasistatic problems, Comput. Mech. 38 (2006) 265-281.

[10] A. Prosperetti, Motion of two superposed viscous fluids, Phys. Fluids 24 (1981) 1217-1223. 